\title{
Design and Application of Integrated Meteorological Service System in the Yangtze River Basin
}

\author{
Gang Tian, Jizhu Wang* \\ Wuhan Central Meteorological Observatory, Wuhan, China \\ Email: ^56916071@qq.com
}

How to cite this paper: Tian, G., \& Wang, J. Z. (2019). Design and Application of Integrated Meteorological Service System in the Yangtze River Basin. Journal of Geoscience and Environment Protection, 7, 230-240.

https://doi.org/10.4236/gep.2019.78017

Received: January 4, 2019

Accepted: August 23, 2019

Published: August 26, 2019

Copyright () 2019 by author(s) and Scientific Research Publishing Inc. This work is licensed under the Creative Commons Attribution International License (CC BY 4.0).

http://creativecommons.org/licenses/by/4.0/

\section{Open Access}

\begin{abstract}
The overall design and system functions of the meteorological service integrated service system of the Yangtze River Basin are introduced. The system is based on CIMISS (National Integrated Meteorological Information Sharing Platform) for expansion and construction, integrating key correction forecasts, weather model comparisons, flood predictions, etc. The forecasting technology, starting from the flood control and drought relief of the service basin and the comprehensive dispatch of the reservoir, has realized the monitoring of water and rain conditions, the production of key business products, the production and distribution of integrated service products, and the quality tracking assessment. The application shows that the system can make the forecaster effectively grasp the service focus, improve the timeliness and pertinence of the service, and have a good promotion and application prospect in the basin meteorological service.
\end{abstract}

\section{Keywords}

Service System, Water and Rain Warning, Grid Forecast, Product Production and Distribution

\section{Introduction}

The Yangtze River Basin spans the three major economic zones of eastern, central and western China. The total drainage area is 1.8 million square kilometers, accounting for $18.8 \%$ of China's land area. It is the largest basin in the country's seven major river basins, covering 12 provinces (municipalities). At the same time, it is also one of the regions with relatively high precipitation variability and frequent occurrence of droughts and floods in China. It has Three Gorges, Gezhouba and Jinshajiang cascade reservoirs. The basin meteorological service sys- 
tem can provide regulated and targeted hydrological weather forecasting service products for watershed management agencies, key water conservancy hubs and local governments. It is very effective in ensuring the smooth implementation of flood control and drought relief and reservoir optimal dispatching in the Yangtze River Basin. This work has practical significance. In recent years, through the cooperation of hydrometeorological departments, the construction of the river basin meteorological service system has made great progress. For example, the Huaihe River Hydrology Bureau has established the Huaihe River Flood Control Meteorological Information Business System (Bi et al., 2011), the Three Gorges Corporation has established the Three Gorges Reservoir Cascade Dispatching Professional Meteorological Service Business System (Fu et al., 2009) and the Chengdu Meteorological Center (Yang et al., 2012), and the Yangtze River Basin Meteorological Center has built a meteorological and hydrological information sharing platform for the Yangtze River Basin (Wang \& Yang, 2014) (hereinafter referred to as the basin sharing platform), but the above systems are biased towards meteorological hydrological data and information. The collection and sharing, in the monitoring and early warning of hydrometeorological disasters, the application of the flood weather model and the refined grid correction technology, the rapid production and real-time evaluation of meteorological service products, still fail to achieve organic integration, especially in various services. In the production of products, there are deficiencies in the comprehensive processing and analysis of the system and the interaction with the forecasters.

The Yangtze River Basin Meteorological Service Integrated Business System (referred to as "YRB-MSIBS", the same below) introduced in this paper is a watershed meteorological service business system based on the B/S structure developed by the Yangtze River Basin Meteorological Center on the basis of meteorological information sharing in 12 provinces and cities in the basin. The YRB-MSIBS collects hydrological meteorological disaster monitoring and forecasting. The functions of rapid production and release of watershed meteorological service products and quality inspection of key service products are the main support systems for the daily business of hydrometeorological forecasting stations of the Yangtze River Basin Meteorological Center. The rest of the paper will introduce the basic situation, key technologies implementation, functional design and application of the YRB-MSIBS.

\section{Basic Situation of the System}

The YRB-MSIBS focuses on the production and release of comprehensive service products and refined grid business products, and applies the National Integrated Meteorological Information Sharing Platform (CIMISS), Geographic Information System and Local Extended Database, focusing on watershed forecasting services. It provides monitoring and forecasting warning, grid forecasting correction, product production and release, forecasting inspection and evaluation, and can provide product production support for watershed prediction. The river 
basin sharing platform developed by the Yangtze River Valley Meteorological Center has different purposes for data collection and sharing. The main process structure of the YRB-MSIBS is shown in Figure 1.

The YRB-MSIBS uses MyEclipse as the main development tool, JAVA and JS as the main programming language, Open Layers based open source GIS framework, deployed in the Tomcat7.0 Web application server. The main source of data is the National Integrated Meteorological Information Sharing Platform (CIMISS) and the National Water and Rain Information Website. Based on the characteristics of multi-source heterogeneity of hydrometeorological data, the local hydrometeorological oracle system database was established by CIMISS.

\section{Key Technologies for System Implementation}

\subsection{Data Storage Management and Information Sharing}

The YRB-MSIBS's data storage management and information sharing still use the existing system structure and functions of the river basin sharing platform. However, the storage management is based on CIMISS for expansion construction. In the database, the original real-time library and historical database are added with external water and rain data. This product adds new product data. Storage management mainly provides data processing and storage, data outbound management, data backup and recovery, data clearing, and data storage monitoring statistics. The information sharing part is based on the needs of forecasters to carry out grid forecasting correction, product production and release, and forecast product inspection. The basin climate background and real-time meteorological hydrological information are necessary information for forecasting and analysis. We integrate content on the shared platform and add the above information to the forecaster's needs.

\subsection{Correction of Grid Precipitation Forecast}

Based on the numerical forecast products, the watershed precipitation refinement grid forecasting is based on the batching method developed by the Yangtze River Valley Meteorological Center (Zhang et al., 2012), artificial neural network (Li \& Wang, 2003), multi-mode integration and other methods to objectively correct the numerical forecast. The WEBGIS manual interaction method is subjectively revised, and through the various correction techniques, the precipitation grid correction forecasting business products are formed, and the corresponding service intermediate products such as the basin quantitative precipitation forecast and the surface rainfall forecast are automatically generated, and the products are produced by the basin comprehensive service product. The main source of the required intermediate forecast products is published. The YRB-MSIBS's data flow is shown in Figure 2.

The watershed boundary can be selected according to the watershed responsibility division of each business unit, and the numerical forecast data is accessed through a unified data interface. 


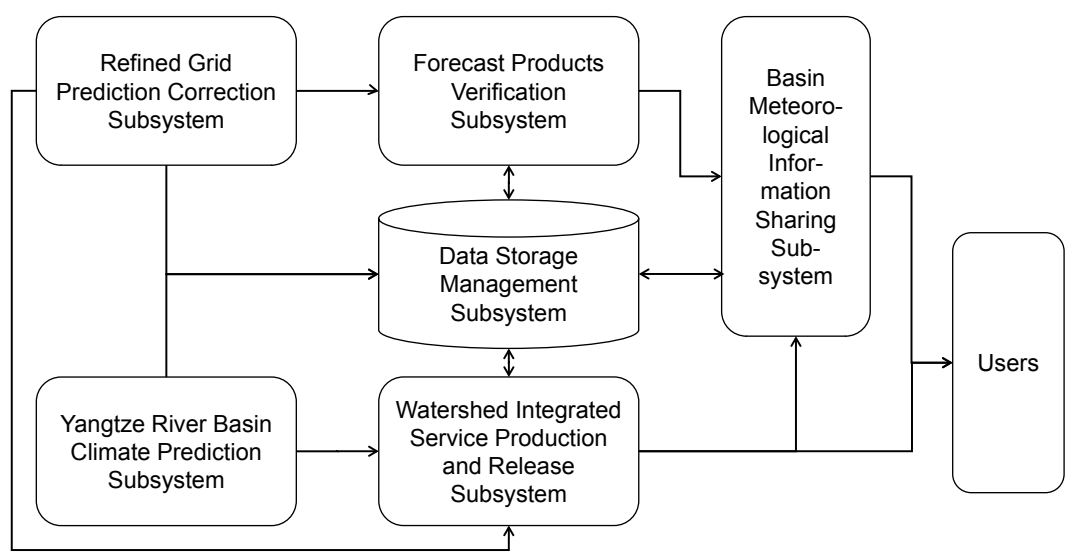

Figure 1. Flow chart of the YRB-MSIBS.

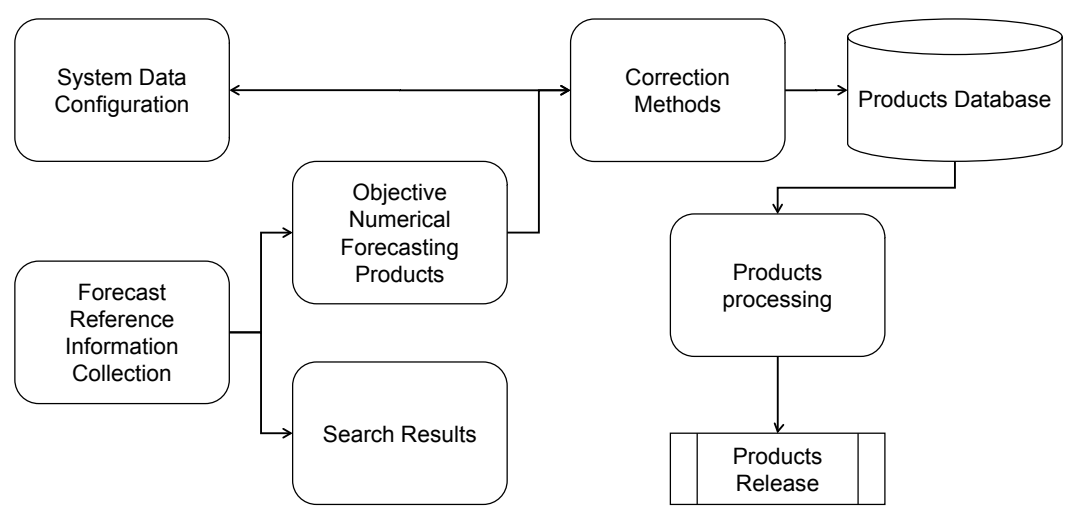

Figure 2. Refined grid forecasting revised data flow chart.

\subsection{Product Production and Distribution}

The production and release of integrated service products is to solve the rapid generation and release of external service comprehensive document products, and to use the newly developed watershed precipitation refined grid forecast correction to generate basin quantitative precipitation forecast and surface rainfall intermediate products, and to use other short, medium and long-term products. Products generated by various types of forecasting key technology systems, using data embedding technology to quickly generate comprehensive service products such as real-time monitoring of watersheds, short-term and medium-term forecasts, and watershed climate predictions, and realize multi-users of service products through multiple communication interfaces. Multi-channel quick release.

\subsection{Forecast Product Inspection}

The forecast product inspection is mainly to carry out quality inspection on the basin precipitation forecast. The inspection content is the surface forecast of the final subjective correction forecast and the model objective forecast. The two test methods of business inspection and service inspection are adopted. The business inspection is carried out according to the requirements of the "National Seven 
Rivers Basin Surface Rainfall Monitoring and Forecasting Business Regulations (Trial)", and the error test and fuzzy score are used to test the surface rainfall forecast quality. The service inspection shall be carried out according to the assessment requirements stipulated by the service unit. The scoring method required by the Three Gorges Corporation is currently provided for the quality inspection of the surface rainfall forecast. The system flow is shown in Figure 3.

\section{Feature Design}

The design of the YRB-MSIBS is mainly based on the rapid analysis of the forecasters and the rapid development of the working environment of the river basin service products. The manual pre-set rules and the system automatically judge the response, and automatically compare the forecast service rules or production scheduling procedures to achieve the work. It also features smart reminders, automatic product creation and one-click service distribution. Strive for forecasters to use the platform to monitor and forecast alarms, grid forecast correction, product production release, forecast inspection and evaluation four modules, keenly grasp the service focus, quickly produce service products, one-click distribution service products and real-time evaluation of service quality.

\subsection{Monitoring and Forecasting Alarm}

In response to the key periods of the major rivers and key reservoirs in the Yangtze River Basin, such as the fallout period, flood season and water storage period, the monitoring and warning of water and rain conditions and forecasting alarms will be provided to enable forecasters to make timely changes in water and rain conditions while completing daily routine product production. Produce non-scheduled products such as rain newsletters and important weather reports to carry out tracking services.

1) Water condition monitoring and alarm. In combination with the National Defense General, the Yangtze River Water Resources Commission and the dispatching regulations of the key reservoirs, when the water level of the main rivers exceeds the over-defense, super-alert, super-guarantee, or the water level of the key reservoir exceeds the limit, the super-design, and the water level of the hydrological control station When the increase exceeds the preset value; when the main river hydrological station flow exceeds the flood control flow, or the control flow set by the key reservoir super-scheduling regulations, and the flow increase exceeds the preset value, the system automatically flashes on the GIS map. Perform water level and flow alarms. At present, real-time monitoring of 516 rivers and rivers hydrological control stations and 186 large and medium-sized reservoirs in the Yangtze River is provided.

2) Rain monitoring and alarm. The single-station rainfall or the main tributary rainfall is over the preset threshold, and the station and the basin area automatically flash to perform the site rainfall and surface rainfall alarms. When the 


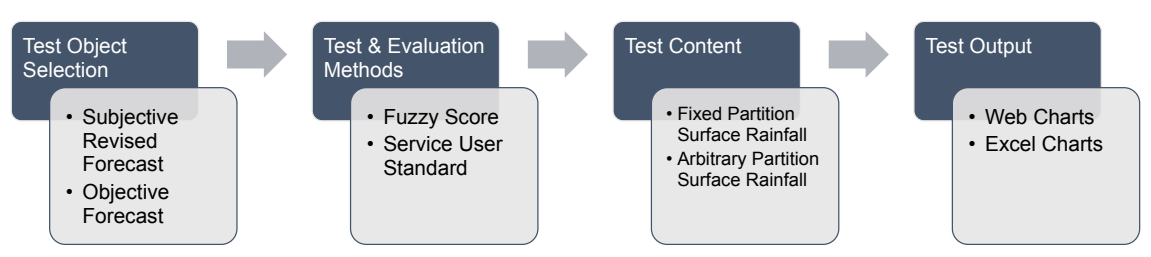

Figure 3. Flow chart of forecast products test system.

daily rainfall is greater than $20 \mathrm{~mm}$ or the multi-station daily rainfall reaches the set threshold, the system automatically reminds the forecaster to produce products such as the "Yangtze River Valley Rainstorm Express". The system screened 721 national meteorological stations to participate in the monitoring of rainfall in the basin, and provided real-time monitoring of the rainfall of 87 basins in the five types of zoning schemes.

3) Overview of water and rain conditions and real-time historical data statistics. The system automatically generates watershed water rain summary text and list information; it can provide water level, flow water information and river basin rainfall, river surface rainfall information, GIS query and histogram, line chart, process line and other charts. Formal query and image list data output; provide water rain history historical ranking information query; provide custom monitoring information for the generation of various types of products such as stain maps and contours. The key hydrological station and national weather station data use the climatic data statistics from 1980 to 2010 as the climatic average, which can be used for seamless query from 1981 to the current data.

4) Surface rainfall forecast alarm. Provide real-time comparison of objective numerical forecast or subjective forecast of main tributary rainfall in the Yangtze River, and the surface rainfall exceeds the threshold to alarm. For example, if the surface rainfall of the main tributaries in the upper reaches of the Yangtze River exceeds $70 \mathrm{~mm}$ or the surface rainfall of the two basins exceeds $50 \mathrm{~mm}$, the system automatically reminds the forecasters to consider the "Important Meteorological Report of the Yangtze River Basin".

5) Flood forecasting and warning. The flood forecasting method for the pilot study of hydrometeorological services in the Yangtze River Basin (Jin et al., 2012) provides flood forecasting results for the upper reaches of the Yangtze River based on real-time flow and surface rainfall forecasting. The system provides real-time estimation of the upper reaches of Minjiang, Jialingjiang, Wujiang, Yibin to Chongqing, Chongqing to Yichang, and provides flood forecasting and warning.

The system timely warned the 24-hour rainfall forecast for the Three Gorges interval on June 30, 2016 and the real-time water regime of the river on July 6, so that the forecasters can grasp all kinds of objective and their own subjective forecasts in time. The impact and the real-time dynamics of river and river rains supported the prediction of a good flood peak in the upper reaches of the Yangtze River and a flood peak in the middle reaches. 


\subsection{Grid Prediction Product Production}

Head Establish a correction platform based on webgis, which can superimpose grid point and non-grid point data, and correct the grid point data. It provides grid-based numerical forecasting, batching method, artificial neural network, multi-mode integration and other objective correction grid point forecast data and empty data and other types of grid point data import functions. Provides grid production of 0 - 10 days, 3 hours interval and above, and provides automatic generation of multi-format products such as grid contour forecast, image data for surface rainfall forecast, micaps format file and database.

In order to improve the correction efficiency of grid data, the system provides the linkage comparison display of forecast products such as EC, Japan, and central meteorological observatory forecast in the eagle-eye mode, providing regional correction, brush and other grid points to quickly correct operations, and The correction process realizes real-time watershed surface rainfall linkage calculation. The system can provide superimposed display of various micaps format data, and can quickly refer to important weather models such as small flood weather model in the basin (Wang et al., 2015) and upstream and downstream flood encounter weather model (Zhang et al., 2015; Chen et al., 2015), which can be used as auxiliary analysis for basin precipitation forecast correction.

Grid point manual correction assignment rule: The artificial interaction area correction adopts the "gradient modification" method that can preserve the objective prediction of the original rain area shape. When the specified area is selected for modification, the system will follow the spatial distribution of the original data. In the case of changing the shape of the rain zone, the precipitation value is adjusted for the zone value. Firstly, according to the selected area, the system calculates the maximum rainfall point in the numerical model forecast in the area. The point is centered until the edge of the area, and the values are successively decreased until it is reduced to the lower limit of the required precipitation level (Figure 4).

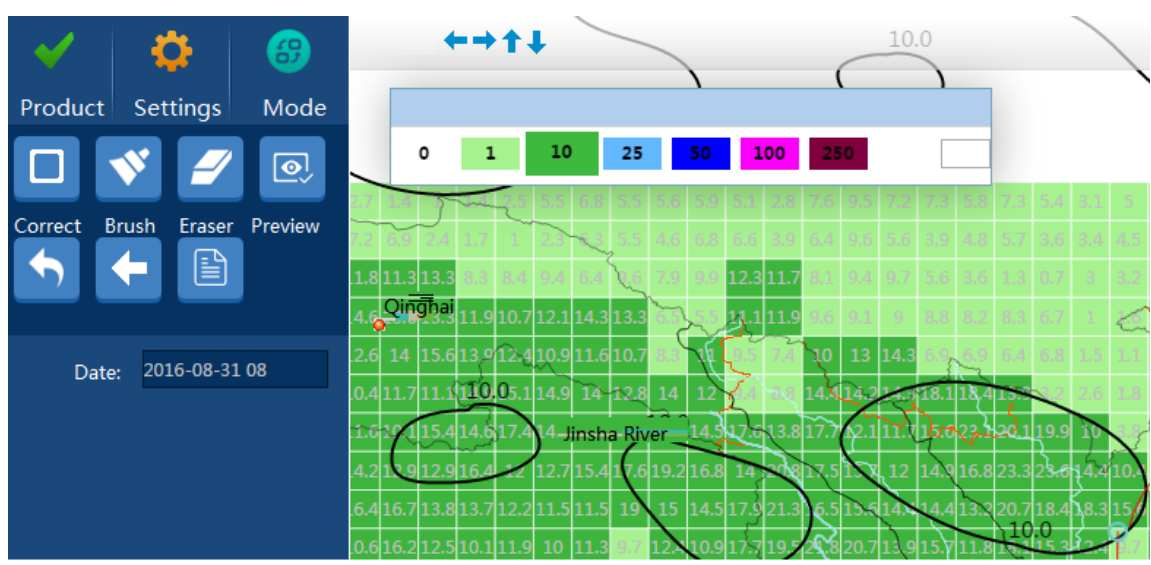

Figure 4. Relevant screen shots of river basin precipitation refinement grid forecasting correction page. 


\subsection{Service Product Production and Distribution}

1) Product production. Create a production platform based on automatic embedding of templates, and design templates for partition blocks to be edited and automatically imported and interactively produced. After the production is started, the automatic insertion of the label content is realized, the data chart generated by the automatic statistics is embedded, the corresponding statistical text and the production time are inserted; before the product is saved, the product can be edited, and the corresponding text and chart can be freely added. It can also provide a preview of the effect of the product; provide key information prompts (mainly the weather tips of the main focus) for interactive production and storage, and the corresponding information can be referenced on the first page of the system or by other system pages; You can add service products as needed, and set up thresholds to monitor and alert the product. For example, after a production and forecast of the "Yangtze River Basin Weather Bulletin", after the production is started, the system extracts data from the CIMISS library, automatically generates a live description text of the rain, automatically generates a map of imported live precipitation and basin surface rainfall, and automatically imports it. After the correction of the 3-day precipitation and the basin surface rainfall forecasting speckle map, the forecaster's work is only for editing the 3-day forecast text description and information collation task. At present, the Yangtze River Valley Meteorological Center has announced the "Yangtze River Basin Weather Bulletin", "Yangtze River Basin Forecast", "Yangtze River Basin Rainfall Forecast", "Yangtze River Basin Rainstorm Express", "Yangtze River Flood Control and Drought Emergency Meteorological Service Materials", etc.

2) Product distribution. It can set the corresponding publishing object and publishing channel for each business product and service product, and provide one-click publishing of multiple service objects to different communication interfaces, which can realize multiple channels such as email, ftp, notes, remote directory, fax, etc. The mode is sent, and the status monitoring of the success or failure of the product to each object is returned.

The system provides a model of small flood weather in the basin and a weather model for upstream and downstream floods. It provides users with service requirements, service content, and service points to facilitate the forecasters to provide service conclusions in conjunction with current service priorities.

\subsection{Forecast Product Inspection}

The system provides objective numerical forecasts such as EC, japan, t639, central grid point forecasting, and provides locally published grid-corrected products and inspection and evaluation of forecast quality of forecasters. It can track the objective and subjective forecasts of each model in the basin. The pros and cons of precipitation forecast; provide error test of forecast and reality, scoring of fuzzy scoring method of China Bureau assessment, and quality score of strong precipitation forecast of service unit assessment. In terms of expressions, the 
comparison of live and forecast, multi-objective forecast and subjective joint comparison in the form of tables, line graphs, and histograms can provide monthly, quarterly, and yearly setting of watershed partition scores.

\section{Application}

The YRB-MSIBS was officially launched in the Yangtze River Valley Meteorological Center in April 2016. Currently, the production and distribution of medium and short-term forecast service products have been switched to the system. The system has the following advantages: 1) High degree of automation. The system has realized automatic processing from the monitoring, forecasting, early warning, production, release and evaluation of the watershed service, which greatly reduces the manual operation and realizes the management of the residue. The design based on $\mathrm{B} / \mathrm{S}$ is simple to deploy and flexible in operation, suitable for hydrometeorology. The forecasting service business focuses on the current situation of a wide area and a small number of people. 2) Strong integration with hydrological business. The system can be combined with the National Defense General, the Yangtze River Basin Water Conservancy Commission and the dispatching procedures of the key reservoirs to provide automatic response to real-time and forecasted water level and real-time thresholds and incremental thresholds. It can be combined with flood forecasting professional forecasting models and flood weathering. The feature model provides flood forecast support. The newly developed 10-hour refined grid forecast product integrates the advantages of numerical forecasting and manual correction, and extends the weather forecasting time required for flood forecasting to more than 3 days, which can effectively improve the accuracy and timeliness of flood forecasting. 3) Professional forecasting services have been applied. In 2016, the system has applied the meteorological services of the Yangtze River Basin and the meteorological services of the Three Gorges and Jinshajiang Reservoirs. The frequency and accuracy of the products have been effectively improved, which has played a good role in accurately predicting the two flood peaks in the Yangtze River Basin in early July. At present, the system's grid correction forecasting subsystem has been transplanted to the Three Gorges Ladder Communication Center, and other river basin meteorological centers such as the Yellow River, Haihe River and Pearl River have also applied localized applications.

\section{Conclusion}

The YRB-MSIBS is based on the CIMISS data storage structure for expansion construction, and fully applies the information collection and sharing functions of the river basin sharing platform, which reduces the difficulty of system development and maintenance. From the requirements of flood control and drought relief and reservoir dispatching to meteorological requirements, combined with hydrological dispatching regulations, water rain monitoring and alarming, production of key business products, production and distribution of 
comprehensive service products and quality tracking assessment are realized, which is conducive to the rapid grasp of forecasters, which is conducive to the key and focus of the basin service that forecasters can quickly grasp.

The YRB-MSIBS integrates a number of key forecasting techniques such as grid correction forecast, weather model comparison, flood forecasting, etc. The business service products realize the automatic embedding of data information, realizing the rapid one-button release of multi-service objects of classified products. The objective and subjective forecasting comparison evaluation was realized, and the system intensive of the key supporting technology for product production was realized. The application of YRB-MSIBS shows that the system is simple to deploy, flexible in operation, functional design of work intelligent reminder, automatic product production, and one-click service distribution, which can greatly reduce the workload of forecasters, and the timeliness and pertinence of services are effectively improved. It has a good prospect of popularization and application in watershed meteorological services.

To highlight pathway for future research, the post-system should utilize high-performance distributed computing and machine learning technology, taking into account the intelligent, automated and rapid production work scenarios of forecast service products and the information fusion analysis and evaluation application scenarios of meteorological impact on the industry, and further improve the intelligence of the system platform, automation, modularization, and customizable level; the focus is to match the location information of the affected factors through weather forecasting, and provide intelligent and optional weather decision suggestions for travel schedule, production scheduling and disaster prevention resource allocation.

\section{Conflicts of Interest}

The authors declare no conflicts of interest regarding the publication of this paper.

\section{References}

Bi, S., Li, L., Cheng, F., \& Liang, J. (2011). Analysis and Suggestions on the Status Quo of Meteorological Services in the Huaihe River Basin. Science and Technology Management Research, 11, 106-109.

Chen, W., Zhang, P., Tian, G., Dong, L., Wei, H., Xu, W., Yue, Y., \& Che, Q. (2015). Analysis of the Classification Characteristics of the Floods in the Upper Reaches of the Yangtze River. Resources and Environment of the Yangtze River Basin, 24, 2124-2152.

Fu, X., Fan, H., Yuan, X., Zuo, X., Liu, W., \& Chen, L. (2009). The Meteorological Service Business System of the Three Gorges Reservoir Cascade Dispatching. Hydropower Energy Science, 27, 1-4.

Jin, Q., Wang, J., Gao, Q., Meng, Y., \& Chen, L. (2012). Flood Prediction System and Experimental Evaluation of the Three Gorges Reservoir Based on Flood Forecasting. Disaster Science, 27, 54-58.

Li, C., \& Wang, R. (2003). Short-Term Heavy Rainfall Forecast System in the Upper 
Reaches of the Yangtze River. Meteorology, 29, 34-37.

Wang, H., \& Yang, D. (2014). Design and Implementation of Meteorological and Hydrological Information Sharing System Based on CIMISS in the Yangtze River Basin. Anhui Agricultural Sciences, 42, 11565-1157.

Wang, H., Li, B., Li, Z., Li, Y., \& Li, J. (2015). Classification and Characteristics of Small and Medium Floods in the Upper Reaches of the Yangtze River. Heavy Rain Disaster, 34, 293-301.

Yang, D., Qin, Y., Wang, H., \& Gong, X. (2012). Professional Meteorological System of Chengdu Tuning Center. Computer System Application, 21, 106-110.

Zhang, P., Long, L., Zhang, N., Deng, H., \& Han, Q. (2012). Application of "Partitioning Method" in Heavy Rain Forecast in Hubei Province. Journal of Tropical Meteorology, 28, 771-776.

Zhang, P., Zhang, J., Tian, G., Xiang, Y., \& Chen, W. (2015). Research on the Conceptual Model of Flood Encounters in the Upper Reaches of the Yangtze River and Dongting Lake. People's Yangtze River, 12, 27-32. 